\title{
Improvement of semen quality by nocturnal scrotal cooling and moderate behavioural change to reduce genital heat stress in men with oligoasthenoteratozoospermia
}

\author{
A. Jung, M. Eberl and W-B. Schill \\ Center of Dermatology and Andrology, Justus-Liebig-University Giessen, \\ D-35392 Giessen, Germany
}

A questionnaire assessing factors that might cause an increase in scrotal temperature was completed by patients with reproducible oligoasthenoteratozoospermia of idiopathic nature or caused by varicocele. Evaluation by means of a grading scale revealed increased scrotal heat stress in oligoasthenoteratozoospermic patients compared with normozoospermic men $(P<0.01)$. In addition, long-term determination of $24 \mathrm{~h}$ scrotal temperature profiles showed that compared with semen donors, oligoasthenoteratozoospermic patients frequently had scrotal temperatures above $35.5^{\circ} \mathrm{C}$ despite the same environmental temperatures $(P<0.05)$. In $88 \%$ of cases, maximum scrotal temperatures were measured during rest or sleep phases, whereas minimum values were recorded during physical activity or frequent change of position.
Nocturnal scrotal cooling by means of an air stream resulted in a decrease in scrotal temperature of approximately $1^{\circ} \mathrm{C}$. Furthermore, a highly significant increase in sperm concentration $(P<0.0001)$ and total sperm output $(P<\mathbf{0 . 0 0 0 1})$ was achieved after nocturnal scrotal cooling for 12 weeks together with a moderate decrease in factors leading to genital heat stress. A significant improvement in sperm motility $(P<0.05)$ and sperm morphology $(P<0.05)$ was also observed, but this improvement was markedly less pronounced than the changes in sperm concentration. This study shows the importance of genital heat stress as a cofactor in fertility impairment in men and indicates nocturnal scrotal cooling as a therapeutic option.

\section{Introduction}

In humans and many other mammals, the testes are located extra-abdominally in the scrotum, and, therefore, are maintained at a lower temperature than the core temperature for optimal stimulation of spermatogenesis (Moore and Quick, 1924). Teleologically, the increased risk of testicular trauma resulting from the extra-abdominal location is offset against improved reproductive capacity. Impaired spermatogenesis due to undescended testis is one of the few established factors for decreased fertility and is thought to result from core temperatures that are too high for initiation and maintenance of spermatogenesis (Moore, 1924; Frankenhuis and Wensing, 1979). In experimental animals (Mieusset et al., 1992) and humans (Shafik, 1991; Mieusset and Bujan, 1994; Moelock, 1995), deterioration of spermatogenesis and azoospermia are induced by an increase in scrotal temperature. However, increases in scrotal temperature by $1^{\circ} \mathrm{C}$ as a result of wearing warm polyester-lined athletic supports did not cause suppression of spermatogenesis, even after several spermatogenesis cycles (Wang et al., 1997).

Treatment recommendations for reducing testicular

Email: Andreas.Jung@derma.med.uni-giessen.de temperature and the resultant improvement in semen quality have been reported in epidemiological studies, particularly for men with idiopathic oligoasthenoteratozoospermia or varicocele (Goldstein and Eid, 1989). These treatments include wearing loose and breathable trousers (Parazzini et al., 1995) and underpants (Tiemessen et al., 1996), avoiding hot baths (Lynch et al., 1986) or saunas (Procopé, 1965; Saikhun et al., 1998), avoiding heat exposure at work (Sas and Szöllosi, 1979; Figà-Talamanca et al., 1992; Thonneau et al., 1997), repeated cold washing of genitals (Davidson, 1954), scrotal cooling by applying ice packs at night (Mulcahy, 1984), wearing a testicular hypothermia device during the day (Zorgniotti et al., 1986) and varicocele operation or sclerosing therapy (Yamaguchi et al., 1989). However, whether such measures have a positive influence on basic semen characteristics or pregnancy rates remains controversial (Buiatti et al., 1984; Levine et al., 1992; Oldereid et al., 1992; Munkelwitz and Gilbert, 1998; Nieschlag et al., 1998).

Although long-term monitoring of patients with varicocele and control volunteers revealed nocturnal scrotal temperatures higher than $36^{\circ} \mathrm{C}$ (Jockenhövel et al., 1990; Lerchl et al., 1993), the influence of the temperatures on spermatogenesis remains to be clarified. Factors such as sleep duration and position, or increased warmth from night clothing or the insulation effect of the blanket have not been 


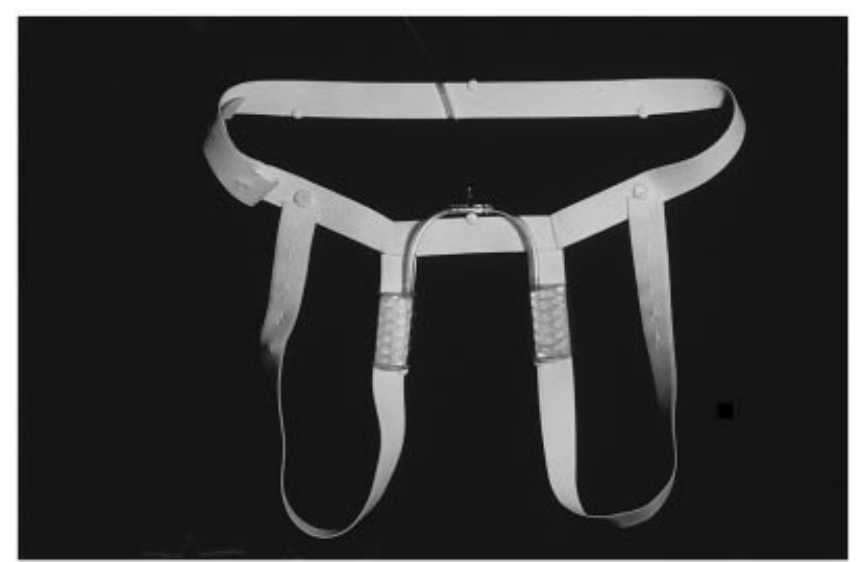

Fig. 1. Cooling device used to reduce scrotal temperatures at night.

considered to date. Similarly, only limited consideration has been given to the importance of physical activity, although significantly lower scrotal temperatures are expected during physical activity compared with those during physical rest, for example, in the sitting or lying position (Brindley, 1982; Lerchl et al., 1993). The dynamic factors influencing testicular temperature have been discussed in several review articles (Mieusset, 1998; Thoneau et al., 1998; Morgentaler et al., 1999).

The present study was performed to determine whether semen quality could be improved by lowering scrotal temperatures by using a simple cooling device (continuous air stream) at night in men with oligoasthenoteratozoospermia of idiopathic nature or caused by varicocele. In addition, a questionnaire was developed to determine and evaluate scrotal heat stress factors (Brindley, 1982), focusing mainly on increased scrotal temperature during sleep (Lerchl et al., 1993) and physical inactivity (Mieusset, 1998). Scrotal temperature profiles were also determined over $24 \mathrm{~h}$.

\section{Materials and Methods}

\section{Equipment}

The cooling device used to reduce scrotal temperature (Fig. 1) was made using an $8 \mathrm{~mm}$ drill. Twenty holes were drilled into one flat side of each of two polyvinyl chloride (PVC) receptacles $(30 \mathrm{ml}$ sample bottles, $64 \mathrm{~mm} \times$ $40 \mathrm{~mm} \times 17 \mathrm{~mm}^{3}$ ). Contralaterally, the receptacles were fixed on an elastic band $30 \mathrm{~mm}$ in width, one side of which was sewn on to an abdominal belt. The distance between the receptacle and the upper border of the belt was $60 \mathrm{~mm}$. The distance between the inner borders of the elastic bands at the belt was $90 \mathrm{~mm}$. The elastic bands were placed around the thighs and were fixed at the belt, and the receptacles were situated in the groin with the openings facing the scrotum. A continuous air stream was supplied through a plastic tube (approximately $6 \mathrm{~m}$ in length, inner diameter $4 \mathrm{~mm}$, outer diameter $6 \mathrm{~mm}$ ) connected to a membrane pump (Schego optimal, $5 \mathrm{~W}, 250 \mathrm{I} \mathrm{h}$; ; Schemel \& Goetz GmbH \& Co. KG, Offenbach/Main). Owing to the mild but still perceivable noise of the pump it was recommended that the pump be placed outside the bedroom. The duration of use of the cooling device was recorded each day by the patients.

For continuous measurement of scrotal temperature over two nights (comparison between cooling and non-cooling) and determination of a $24 \mathrm{~h}$ temperature profile (Jockenhövel et al., 1990), temperature sensors (YSI model 409BD; Yellow Springs Instrument Co., Yellow Springs, $\mathrm{OH}$; accuracy of measurement $\pm 0.1^{\circ} \mathrm{C}$ ) were fixed on either side of the scrotum using Fixomull ${ }^{\circledR}$ stretch (30 $\mathrm{mm} \times 30 \mathrm{~mm}$; Beiersdorf AG, Hamburg) throughout the monitoring period. Values were registered every $2 \mathrm{~min}$ via a portable data recorder AGF-Thermoport 2 (Funkelektronik Gräwe, Münster). In parallel with measurement of scrotal temperatures, patients and volunteers recorded the type of activity they were undertaking, including bed rest, sitting, standing and physical activity as well as a mixture of all these types of activity.

\section{Selection of patients for cooling treatment}

Between September 1998 and September 1999, patients with oligoasthenoteratozoospermia of idiopathic nature or caused by varicocele (assumed pathogenetic role of increased genital heat stress) attending the andrological outpatient department were invited to participate in a therapeutic study for improvement of semen quality using nocturnal scrotal cooling for 12 weeks. Approval to perform the study was obtained from the Ethic Commission at the Faculty of Human Medicine, Justus Liebig University Gießen (application 35/98) with an amendment on September 1998. All patients gave written informed consent to participate in the study. The patients showed no signs of testicular damage (normal gonadotrophin concentration, testicular volume of at least $12 \mathrm{ml}$ on both sides, no retractile testis or history of maldescendent testis), and infection or chronic inflammation of the seminal tract was excluded. Varicocele was diagnosed clinically and by using Doppler sonography. During the 3 months before the study, no therapy had been performed to improve semen quality. A recruitment period of 1 year was chosen to avoid seasonal influences on semen quality (Levine, 1996). Of the 25 patients ('potential patients undergoing cooling treatment') who were initially interested, 20 participated in the study ('patients undergoing cooling treatment'). The remaining five men withdrew from the trial during preliminary examinations.

The influence of scrotal cooling on semen quality and serum hormone status was assessed in the 20 patients undergoing cooling treatment. At least one previous spermiogram with oligoasthenoteratozoospermia was followed by an initial spermiogram at the beginning of the trial (oligoasthenoteratozoospermia as inclusion criterion) according to the World Health Organization (1992) and 
Table 1. Questionnaire for scoring individual scrotal heat exposure during 3 months

\begin{tabular}{|c|c|c|c|}
\hline & \multicolumn{3}{|c|}{ Points } \\
\hline How many hours per day do you sleep? & \multicolumn{3}{|c|}{2 per h } \\
\hline \multicolumn{4}{|l|}{$\begin{array}{l}\text { How many hours per day (apart from sleep) do you spend at physical rest } \\
\text { (sitting, standing, lying) while eating, working, watching TV, driving or travelling by train? }\end{array}$} \\
\hline & Yes & Partly & No \\
\hline \multirow{2}{*}{$\begin{aligned} & \text { During the day do you wear: } \text { tight underpants (for example, jockey)? } \\
& \text { tight trousers (for example, jeans)? }\end{aligned}$} & 4 & 2 & 0 \\
\hline & 4 & 2 & 0 \\
\hline \multirow{3}{*}{$\begin{array}{l}\text { At night do you wear: underpants? } \\
\text { pyjamas? }\end{array}$} & 2 & 1 & 0 \\
\hline & 2 & 1 & 0 \\
\hline & Yes & & No \\
\hline Do you use a down-filled duvet? & 2 & & 0 \\
\hline Do you use saunas? & 2 & & 0 \\
\hline Do you take hot baths? & 2 & & 0 \\
\hline Do you use a heated blanket? & 2 & & 0 \\
\hline Do you use a heated seat in your car? & 2 & & 0 \\
\hline Are you exposed to heat at work? & 2 & & 0 \\
\hline Have you had a fever in the past 3 months? & 2 & & 0 \\
\hline Do you use a solarium? & 2 & & 0 \\
\hline Score value: & \multicolumn{3}{|c|}{ Total points } \\
\hline
\end{tabular}

serum concentrations of $\mathrm{FSH}, \mathrm{LH}$ and total testosterone were determined. Follow-up spermiograms were analysed after 4, 8 and 12 weeks (deviation of \pm 1 week was allowed). The serum hormone status was redetermined at the end of the trial after 12 weeks.

\section{Questionnaire assessing scrotal heat exposure and description of control group 1}

Factors that might have a negative influence on scrotal temperature were assessed by means of a questionnaire to clarify whether all 25 potential patients undergoing cooling treatment had increased genital heat stress. Both the questionnaire and evaluation by means of a grading scale (Table 1) were designed to record the influence of physical inactivity (high scrotal temperature during sleep or when sitting) through a time-dependent multiplier. Clothing habits and coincidental increases in temperature as a result of fever or lifestyle habits were also considered. The questions referred to mean values from the previous 3 months and included the last spermatogenesis cycle.

The results from the questionnaires of the 25 potential patients undergoing cooling treatment were compared with data from 26 normozoospermic men with sperm concentrations $>10^{8} \mathrm{ml}^{-1}$ (control group 1 ; infertile wife) who had been interviewed during the same period. At the end of the study, the questionnaire was repeated by the 20 patients undergoing cooling treatment to determine the influence of behavioural change in addition to nocturnal scrotal cooling.

\section{Scrotal temperature profiles and description of control group 2}

Because of the limitations of the questionnaire, the $24 \mathrm{~h}$ scrotal temperature profiles of the patients undergoing cooling treatment were compared with those of ten semen donors (fertile or normozoospermic, control group 2). Long phases of sleeping, sitting or moving were noted. In addition, the outdoor temperatures measured each hour by the Gießen weather station (Deutscher Wetterdienst, Offenbach) were considered to exclude all environmental influences of temperature. After the $24 \mathrm{~h}$ scrotal temperature profile was recorded, the patients undergoing cooling treatment continued to measure scrotal temperature during the subsequent night. The cooling device was used to evaluate the individual cooling effect compared with the first night without cooling.

\section{Patients with oligoasthenoteratozoospermia without cooling as control group 3}

As deviating semen parameters were unavoidable and it was impossible to perform a prospective double-blind randomized trial on the effect of scrotal cooling, the spermiograms of another 20 patients with oligoasthenoteratozoospermia of idiopathic nature or caused by varicocele (normal gonadotrophin concentrations, testicular size at least $12 \mathrm{ml}$ on both sides, no retractile testis or history of maldescendent testis; control group 3) were evaluated at the same time, during which no therapy was performed because of current diagnostic examination. The first semen 


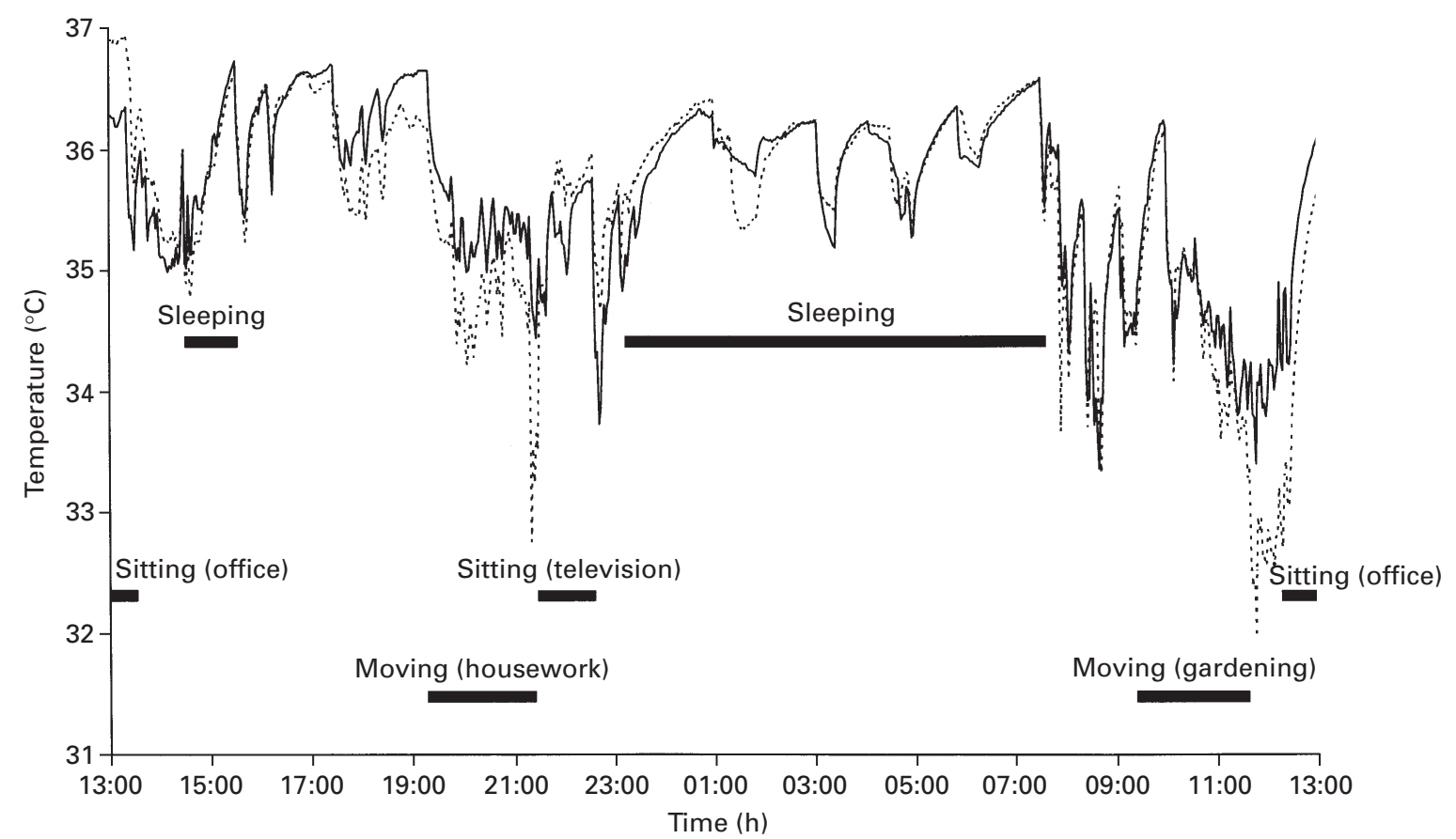

Fig. 2. Scrotal temperature profile over $24 \mathrm{~h}$ in a single patient with oligoasthenoteratozoospermia at an average outdoor temperature of $17.8^{\circ} \mathrm{C}$ and record of activity during the $24 \mathrm{~h}$ (right scrotum (-), left scrotum (-------)). Temperature curves depend on the degree of activity: maximum values were obtained during physical rest and minimum values during physical activity.

analyses of control group 3 were performed as close as possible to week 0 of analysis of a cooling patient (longest interval was 2 weeks). The second semen analyses of control group 3 were evaluated at intervals between weeks 7 and 16. This range was chosen because it represents the minimum duration for the 8 week analysis of patients undergoing cooling treatment (deviation of \pm 1 week was allowed) and the maximum duration of the 12 week analysis of patients undergoing cooling treatment.

\section{Statistical analysis}

Tests for non-parametrically distributed values only were used. The $P$ values were calculated two-sided. The Mann-Whitney $U$ test was used for comparison of the scores for heat stress among patients undergoing cooling treatment and control group 1 . The scores before and after cooling were compared using Wilcoxon paired sample test. The Mann-Whitney $U$ test was applied for comparison of temperature frequencies above $35.5^{\circ} \mathrm{C}$ in patients undergoing cooling treatment and control group 2. Comparison of the median values of the nocturnal scrotal temperatures in patients undergoing cooling treatment, with and without cooling, was made using Wilcoxon paired sample test. Basic spermiogram parameters (concentration, motility, morphology) and serum hormone concentrations of the patients undergoing cooling treatment were analysed using Wilcoxon paired sample test and together using Friedman's test for repeated measurements. Changes in the semen analyses of control group 3 were compared using Wilcoxon paired sample test.

\section{Results}

The questionnaires (Table 1) assessing the possible influence of heat on spermatogenesis in patients with oligoasthenoteratozoospermia of idiopathic nature or caused by varicocele (including the five patients who did not participate in the cooling treatment study) and in normozoospermic men with sperm concentrations $>10^{8} \mathrm{ml}^{-1}$ (control group 1) revealed significant genital heat stress in patients with impaired semen quality $(P<0.01$; score values for median and 10-90\% percentile, respectively: 46 versus $36 ; 34-52$ versus $28-48$ ). The higher values were primarily due to longer periods of physical rest (sitting, standing or lying; median: $10 \mathrm{~h}$ versus $8 \mathrm{~h}$ ) independent of night rest (median: $7 \mathrm{~h}$ in both groups). Increased periods of physical rest showed statistical significance $(P<0.05)$. In addition, tight-fitting underwear was worn significantly more frequently $(P<0.02)$.

Long-term monitoring of scrotal temperatures in patients undergoing cooling treatment and semen donors (control group 2) again showed that physical activity is an important factor, which steadily improves scrotal heat dissipation (Brindley, 1982; Lerchl et al., 1993); a representative example of a profile from a cooling patient is shown (Fig. 2). Physical inactivity during desk work or night rest was associated with scrotal temperatures of $>36^{\circ} \mathrm{C}$, whereas 


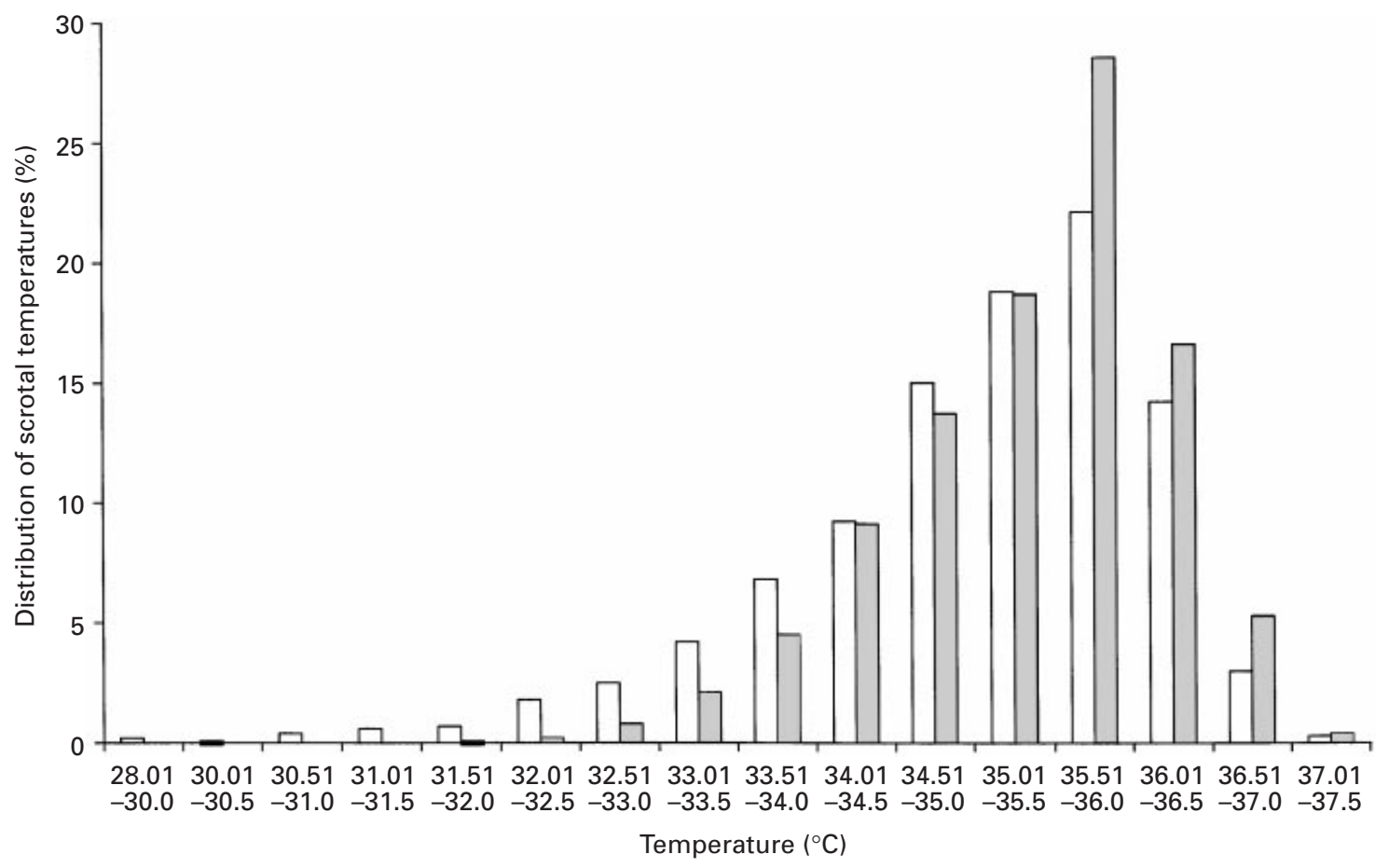

Fig. 3. Distribution of scrotal temperatures in ten semen donors (control group 2; $\square$ ) and ten patients with oligoasthenoteratozoospermia of idiopathic nature or caused by varicocele $(\square)$ at comparable outdoor temperatures. For each individual 1440 temperature values over $24 \mathrm{~h}$ were included. For statistical analysis, the number of temperature values above $35.5^{\circ} \mathrm{C}$ per individual was calculated and was found to be significantly higher in patients with oligoasthenoteratozoospermia than in the control group $2(P<0.05)$. Mann-Whitney $U$ test (twosided).

temperatures between $34^{\circ} \mathrm{C}$ and $35^{\circ} \mathrm{C}$ or lower were measured during housework or gardening.

The $24 \mathrm{~h}$ scrotal temperature profiles of the 20 patients undergoing cooling treatment and the ten semen donors (control group 2) differed significantly in that scrotal temperatures $>35.5^{\circ} \mathrm{C}$ were predominant in patients before nocturnal cooling $(P<0.002)$. Median values for scrotal temperatures during sleep were higher than those for the total $24 \mathrm{~h}$ profiles (patients undergoing cooling treatment at sleep: $35.92^{\circ} \mathrm{C}$ on the right, $35.73^{\circ} \mathrm{C}$ on the left; $24 \mathrm{~h}$ profile: $35.50^{\circ} \mathrm{C}$ on the right, $35.54^{\circ} \mathrm{C}$ on the left; control group 2 at sleep: $35.76^{\circ} \mathrm{C}$ on the right, $35.57^{\circ} \mathrm{C}$ on the left; $24 \mathrm{~h}$ profile: $34.99^{\circ} \mathrm{C}$ on the right, $35.29^{\circ} \mathrm{C}$ on the left). The $24 \mathrm{~h}$ profiles of control group 2 were compared with those of the ten patients undergoing cooling treatment who had been exposed to the same outdoor temperatures during the period of monitoring to exclude a possible influence by environmental temperature. Mean outdoor temperature was $5.47^{\circ} \mathrm{C}$ in the patients undergoing cooling treatment and $5.50^{\circ} \mathrm{C}$ in control group 2 (Fig. 3). High scrotal temperatures were significantly more frequent in the patients before they received nocturnal cooling treatment $(P<0.05)$.

Statements from control group 2 and the patients undergoing cooling treatment regarding the activity undertaken at the time of maximum and minimum values of scrotal temperature profiles revealed that maximum values occurred during sleeping $(40.0 \%$ in control group 2 and $37.5 \%$ in patients undergoing cooling treatment) and sitting $(50.0 \%$ in control group 2 and $42.5 \%$ in patients undergoing cooling treatment), particularly by the end of a period of such activity. However, minimum values occurred during periods of movement $(60 \%$ in control group 2 and $30 \%$ in patients undergoing cooling treatment) or during alternation between moving, lying, sitting or standing $(25 \%$ in control group 2 and $40 \%$ in patients undergoing cooling treatment). Minimum values of scrotal temperature were accompanied by fewer statements on activity and repeatedly occurred between 06:00 and 09:00 h.

A comparison of the nocturnal scrotal temperatures in patients undergoing cooling treatment, with and without cooling, showed a reduction in the median value of scrotal temperature from $35.8^{\circ} \mathrm{C}$ (range $34.6-36.3^{\circ} \mathrm{C}$ ) to $34.9^{\circ} \mathrm{C}$ (range $32.8-35.5^{\circ} \mathrm{C}$ ). The frequency distribution of scrotal temperatures is shown (Fig. 4). Comparison of the median values using the Wilcoxon paired sample test revealed a highly significant difference.

The following diagnoses were established among the 20 patients undergoing cooling treatment who participated in the study: primary infertility (17), secondary infertility (2) and an unfulfilled wish to father a child for 8 months (1), 


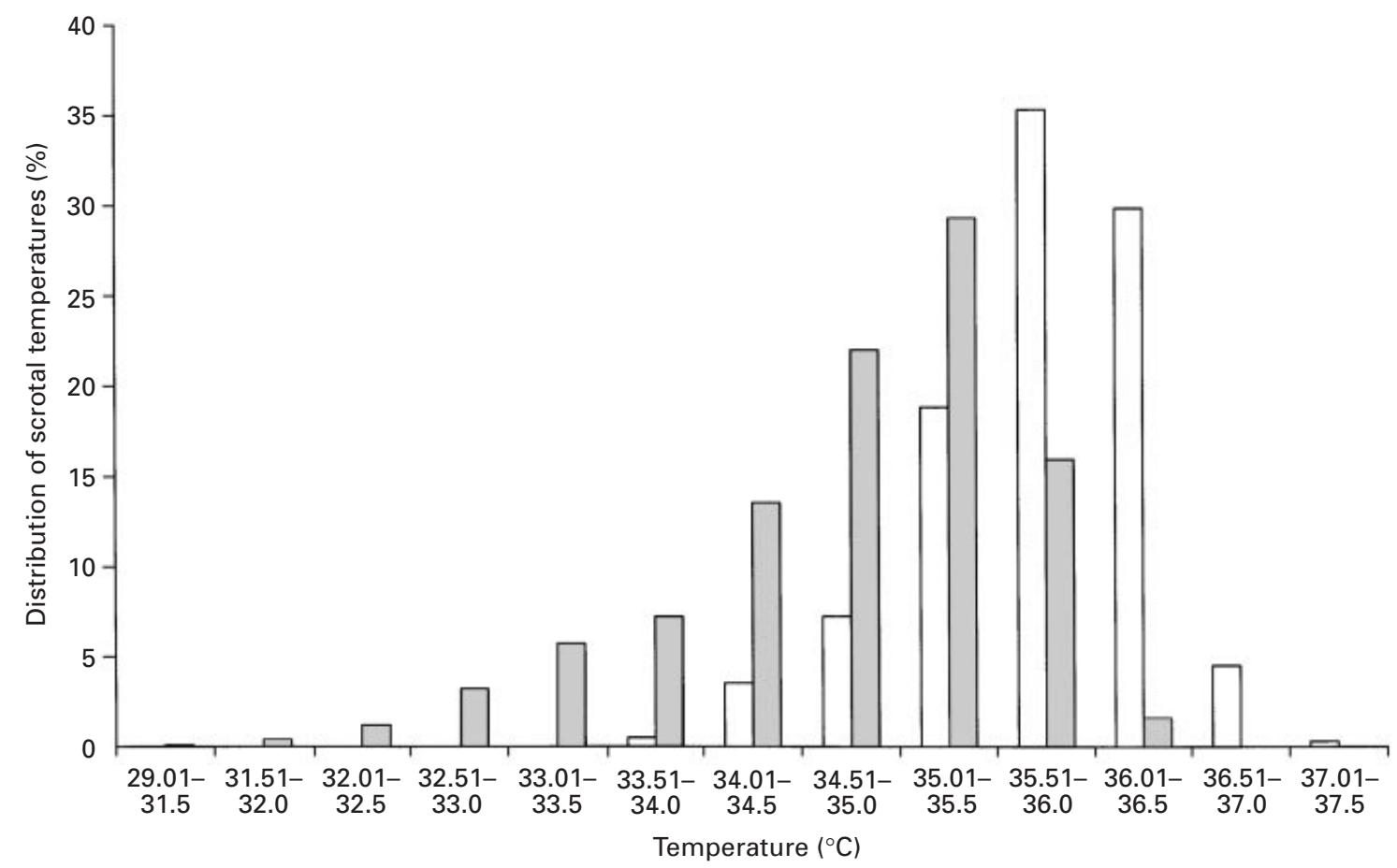

Fig. 4. Duration of scrotal temperatures at night in 19 patients with oligoasthenoteratozoospermia of idiopathic nature or caused by varicocele, without $(\square)$ and with $(\square)$ scrotal cooling. Temperatures above $35.5^{\circ} \mathrm{C}$ were more frequent without cooling. Median values show highly significant difference $(P<0.0001)$. Wilcoxon paired sample test (two-sided).

previously treated for varicocelectomy or sclerotherapy (5, 1 with persisting varicocele), varicocele or subclinical varicocele (4), previously treated for seminal tract infection or inflammation (6), previous anabolic abuse (1) and small prostatic cyst (1). Other relevant parameters of the patients undergoing cooling treatment are summarized (Table 2). One patient deviated widely from the study design (130 days, including 73 nights without cooling) because of poor compliance due to the noise of the membrane pump, which prevented use during overnight accommodation with parents or friends. Other compliance problems with the cooling device were not reported. One patient had a followup semen examination after 8 weeks of therapy, which was performed in another andrological centre.

The semen parameters of the patients undergoing cooling treatment revealed a highly significant improvement in sperm concentration (Fig. 5) and total sperm output (median: $1.99 \times 10^{7}$ before the study, $1.81 \times 10^{7}$ at week $0,3.7 \times 10^{7}$ at week $4,6.86 \times 10^{7}$ at week $8,5.66 \times 10^{7}$ at week 12) (Friedman's test for repeated non-parametric measurements: $P<0.0001)$. In addition, sperm motility (Fig. 6) and sperm morphology (Fig. 7) showed a small, but significant improvement $(P<0.05)$.

The semen parameters of men in control group 3 who did not receive cooling treatment revealed no significant changes in sperm concentration (Fig. 5), motility (Fig. 6) or morphology (Fig. 7).

In the patients undergoing cooling treatment, there was no significant difference in the duration of abstinence (median: 5.5 before the study, 4.5 at week 0, 5.0 at week 4, 5.0 at week $8,5.0$ at week 12) and respective ejaculate volume (median: 4.35 before the study, 3.5 at week 0, 4.0 at week 4, 4.0 at week 8 and 3.75 at week 12).

No change in the concentration of FSH and total testosterone was observed before and at the end of scrotal cooling, but a significant increase in $\mathrm{LH}$ was observed ( $P<0.02$; median: 2.35 versus 3.3 ; and $10-90 \%$ percentile:

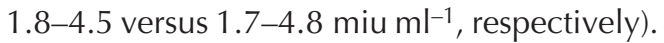

The questionnaire score values of the patients undergoing cooling treatment with regard to genital heat stress at the beginning and end of therapy revealed a significant reduction in factors that influence heat stress. Patients had changed their behaviour, although this had not been requested before the study $(P<0.05$; score values for median and 10-90\% percentile, respectively: 44.5 versus 39; 34-49 versus 28-48). In eight patients, lower scores resulted primarily from fewer rest periods because of increased physical activity during leisure time and in six patients because of less clothing worn at night (no pyjamas or underpants).

\section{Discussion}

In the present study, patients with oligoasthenoteratozoospermia of idiopathic nature or caused by varicocele showed marked scrotal heat stress compared with normozoospermic 
Table 2. Statistical data for further characterization of cooling patients and effective use of scrotal cooling during the study

\begin{tabular}{lrc}
\hline & Median & Range \\
\hline Testicular volume $(\mathrm{ml})$ & 34.5 & $24-50$ \\
Unfulfilled wish to father a child (months) & 43.5 & $8-144$ \\
Number of spermiograms before the study & 4.0 & $2-22$ \\
Number of days with scrotal cooling & 89.5 & $78-130$ \\
Number of days without scrotal cooling & 0.0 & $0-73$ \\
Average duration of cooling per day (min) & 450.5 & $219-535$ \\
\hline
\end{tabular}

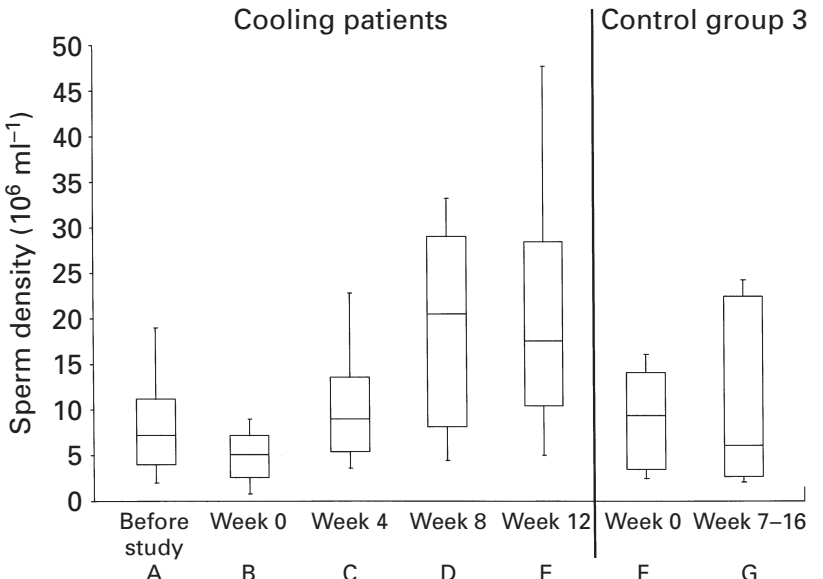

Fig. 5. Influence of nocturnal scrotal cooling on sperm concentration in 20 patients and 20 control patients (control group 3) in which previous spermiograms had repeatedly revealed oligoasthenoteratozoospermia (bars show 10-90\% percentile, columns show $25-75 \%$ percentile and median). Wilcoxon paired sample test, two-sided: column B versus D $(P<0.0001)$, column $\mathrm{B}$ versus $\mathrm{E}(P<0.0001)$, column $\mathrm{F}$ versus $\mathrm{G}(P>0.05)$; and Friedman's test for repeated non-parametric measurements: columns A-E $(P<0.0001)$.

men (control group 1). This finding is supported by the evaluation of score values in questionnaires focusing on genital heat stress. In addition, long-term monitoring of scrotal temperature profiles showed significantly more temperatures $>35.5^{\circ} \mathrm{C}$ in patients before undergoing cooling treatment than in control group 2. There was evidence of an improvement in sperm concentration and total sperm output as a result of nocturnal scrotal cooling and moderate changes in patient behaviour towards genital heat stress, indicating that genital heat exposure should be considered as a causal factor of impaired fertility. Scrotal cooling by means of a continuous air stream represents a simple method for reduction of the relatively high scrotal temperatures observed at night (Jockenhövel et al., 1990; Lerchl et al., 1993). Long-term monitoring of scrotal temperatures in patients before and during use of the cooling device revealed a cooling effect of approximately $1^{\circ} \mathrm{C}$. Scrotal cooling by applying ice bags in the genital region was suggested as a method for improving semen parameters by Robinson et al. (1968), but a study was not

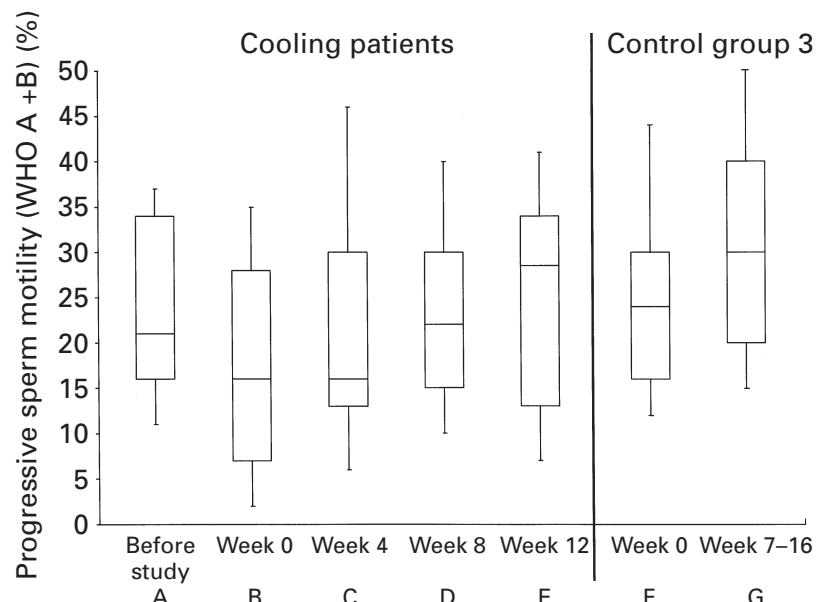

Fig. 6. Influence of nocturnal scrotal cooling on progressive sperm motility $(\mathrm{WHO} A+\mathrm{B})$ in 20 patients and 20 control patients (control group 3) in which previous spermiograms had repeatedly revealed oligoasthenoteratozoospermia (bars show 10-90\% percentile; columns show $25-75 \%$ percentile and median). For patients undergoing cooling treatment, before the study, only 18 values were used because sperm density in two patients was too low for determination of progressive sperm motility. Wilcoxon paired sample test, two-sided: column $\mathrm{B}$ versus $\mathrm{D}(P<0.05)$, column B versus $\mathrm{E}(P<0.05)$, column $\mathrm{F}$ versus $\mathrm{G}(P>0.05)$; and Friedman's test for repeated non-parametric measurements: columns $\mathrm{B}-\mathrm{E}(P<0.05)$.

performed. The first such study was performed by Mulcahy (1984) who used a similar method to investigate the effect of nocturnal scrotal cooling on spermatogenesis. The patients had ice packs applied to the scrotum and held in place with shorts, but scrotal temperatures were not monitored and statistical analysis was limited. Repeated cold washings of the genitals has been recommended as another means of scrotal cooling (Davidson, 1954), but pertinent studies are not available. To date, only an evaporative cooling device for reduction of scrotal temperature has been developed for use in practice (Zorgniotti et al., 1980, 1982, 1986). The cooling effect is achieved by evaporation of water from a body-worn reservoir, which is continuously emitted into a scrotal covering. This cooling device offers the advantage that it can be used during the day in the work environment; however, because of the resultant humid environment the duration of use must be limited to allow regeneration of the scrotal skin. Furthermore, dermatological diseases such as tinea inguinalis or erythrasma are considered contraindications. Among 64 patients (infertile for at least 2 years and partner with normal gynaecological findings) who had worn the cooling device for a minimum of 16 weeks, spermiograms showed improvement in $65.6 \%$ of patients and pregnancy occurred in $26.6 \%$ of cases (Zorgniotti et al., 1986). Overall, patient compliance was only $46 \%$. The study included only patients who had scrotal temperatures above $34.1^{\circ} \mathrm{C}$. This inclusion criterion was established in 


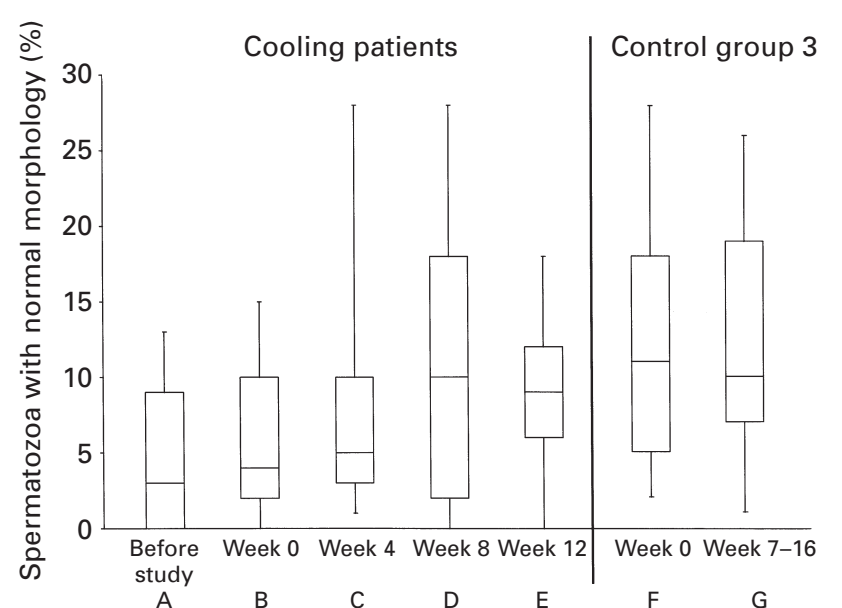

Fig. 7. Influence of nocturnal scrotal cooling on sperm morphology in 20 patients and 20 control patients (control group 3 ) in which previous spermiograms had repeatedly revealed oligoasthenoteratozoospermia (bars show 10-90\% percentile, columns show $25-75 \%$ percentile and median). Wilcoxon paired sample test, two-sided: column $\mathrm{B}$ versus $\mathrm{D}(P=0.165)$, column $\mathrm{B}$ versus $\mathrm{E}(P=0.142)$, column $\mathrm{F}$ versus $\mathrm{G}(P>0.05)$; and Friedman's test for repeated non-parametric measurements: columns B-E $(P<0.05)$.

undressed patients lying on their back in a comfortable environment, and was described as an intrinsic infertility factor in a study by Zorgniotti and Sealfon (1988). The advantage of this technique under standardized environmental conditions is the high interindividual comparability; the disadvantage is that multiple environmental influences, which determine scrotal temperatures under everyday conditions, are not considered. These environmental factors could be assessed more accurately by long-term monitoring of scrotal temperature under everyday physiological conditions. For example, the temperature-reducing effect of frequent physical activity as compared with physical rest has been demonstrated in the present study by evaluating long-term temperature profiles and the patient's history.

A limitation of the present study is that it cannot be performed in a double-blind manner, because the airstream is perceived subjectively. It is not completely satisfactory to evaluate patients with oligoasthenoteratozoospermia of idiopathic nature or caused by varicocele without therapy (control group 3), who do not show a significant change in sperm parameters. Within the framework of intensified andrological therapy, improved semen parameters could also be explained by a marked placebo effect. An argument against this explanation is that the effect is observed after 8 and 12 weeks, but not after 4 weeks. This finding does not support the theory of a placebo effect, whereas the time interval is in agreement with the high susceptibility especially of spermatocytes and spermatids to testicular heat exposure in mammals (Setchell, 1998) and presumably in humans. A placebo effect is also unlikely because increased heat stress was demonstrated in patients in the present study by means of both the questionnaire on genital heat stress and long-term measurement of scrotal temperature. Apart from nocturnal scrotal cooling during the study, patients showed moderate behavioural changes that reduced scrotal heat factors; therefore, the improvement in the spermiograms cannot be attributed solely to scrotal cooling. Another limitation of the study design was that semen parameters were considered, but pregnancy rates were not. However, the study was not designed for this purpose, because a maximum of two ovulations could be expected during the treatment period with improved spermiogram parameters and an additional gynaecological infertility factor was not an exclusion criterion. It would be unethical to perform a study focusing directly on pregnancy rates without having determined a possible effect on spermiogram parameters. Improvement in the spermiogram alone enables many infertile couples to apply simple methods of assisted reproduction, such as intrauterine insemination, and to avoid more invasive and costly reproductive techniques, for example microinjection.

Serum concentrations of FSH, $\mathrm{LH}$ and total testosterone were recorded to determine whether an optimal effect of scrotal cooling resulting in improved spermatogenesis was associated with a slight reduction in FSH concentrations. Similarly, improved testosterone synthesis in the Leydig cells may have led to decreased $\mathrm{LH}$ concentrations; therefore, the increased LH concentrations observed in the present study should not be interpreted as resulting from a significant influence of scrotal cooling.

For further investigation of the influence of scrotal cooling on spermatogenesis, a cooling device that can be worn for $24 \mathrm{~h}$ would be desirable, coupled with a thermosensor to avoid temperature increases beyond a threshold value, for example $35.5^{\circ} \mathrm{C}$. Prospective doubleblind studies could then be performed by setting different threshold values.

Warming of the testis as a method of contraception has been investigated in several studies. It was found that scrotal temperatures increased by $1^{\circ} \mathrm{C}$ as a result of wearing polyester-lined athletic supports, but did not cause suppression of spermatogenesis over several cycles (Wang et al., 1997). However, other studies demonstrated that long-term increases in scrotal temperature as a result of wearing insulating underwear (Rock and Robinson, 1965; Robinson and Rock, 1967) or polyester sling scrotal covers (Moelock, 1995) led to impaired semen quality and azoospermia. Increased scrotal temperatures were also observed in patients wearing a special support that presses the testes close to the inguinal canal (Mieusset and Bujan, 1994) and after surgical fixation of the testes in the inguinal canal (Shafik, 1991). These findings support the results of the present study.

The authors would like to thank G. Haidl for determining the 8 week spermiogram values in a study patient, G. Scharfe for translation of the manuscript, $\mathrm{H}-\mathrm{C}$. Schuppe for helpful discussions and $\mathrm{R}-\mathrm{H}$. Bödeker for valuable hints concerning the statistical evaluation. 


\section{References}

Brindley GS (1982) Deep scrotal temperature and the effect on it of clothing, air temperature, activity, posture and paraplegia British Journal of Urology 54 49-55

Buiatti E, Barchielli A, Geddes M, Nastasi L, Kriebel D, Franchini M and Scarselli G (1984) Risk factors in male infertility: a case-control study Archives of Environmental Health 4 266-270

Davidson HA (1954) Treatment of male subfertility: testicular temperature and varicoceles Practitioner 173 703-708

Figà-Talamanca I, Dell'Orco V, Pupi A, Dondero F, Gandini L, Lenzi A, Lombardo F, Scavalli P and Mancini G (1992) Fertility and semen quality of workers exposed to high temperatures in the ceramics industry Reproductive Toxicology 6 517-523

Frankenhuis MT and Wensing CJG (1979) Induction of spermatogenesis in the naturally cryptorchid pig Fertility and Sterility 31 428-433

Goldstein M and Eid JF (1989) Elevation of intratesticular and scrotal skin surface temperature in men with varicocele Journal of Urology 142 $743-745$

Jockenhövel F, Gräwe A and Nieschlag E (1990) A portable digital data recorder for long-term monitoring of scrotal temperatures Fertility and Sterility $\mathbf{5 4}$ 694-700

Lerchl A, Keck C, Spiteri-Grech J and Nieschlag E (1993) Diurnal variations in scrotal temperature of normal men and patients with varicocele before and after treatment International Journal of Andrology $\mathbf{1 6}$ 195-200

Levine RJ (1996) Male factors contributing to the seasonality of human reproduction Annals of the New York Academy of Sciences 709 29-45

Levine RJ, Brown MH, Bell M, Shue F, Greenberg GN and Bordson BL (1992) Air-conditioned environments do not prevent deterioration of human semen quality during the summer Fertility and Sterility $\mathbf{5 7}$ 1075-1083

Lynch R, Lewis-Jones DI, Machin DG and Desmond AD (1986) Improved seminal characteristics in infertile men after a conservative treatment regimen based on the avoidance of testicular hyperthermia Fertility and Sterility 46 476-479

Mieusset R (1998) Influence of lifestyle on male infertility: potential testicular heating factors Middle East Fertility Society Journal 3 S40-S45

Mieusset R and Bujan L (1994) The potential of mild testicular heating as a safe, effective and reversible contraceptive method for men International Journal of Andrology 17 186-191

Mieusset R, Quintana-Casares P, Sanchez-Partida LG, Sowerbutts SF, Zupp JL and Setchell BP (1992) Effects of heating the testes and epididymides of rams by scrotal insulation on fertility and embryonic mortality in ewes inseminated with frozen semen Journal of Reproduction and Fertility $\mathbf{9 4}$ 337-343

Moelock N (1995) Polyester sling scrotal cover induces oligozoospermia in normal Indonesian men Medical Journal of Indonesia 4 225-230

Moore CR (1924) Properties of the gonads as controllers of somatic and psychical characteristics. VI. Testicular reactions in experimental cryptorchism American Journal of Anatomy 34 269-316

Moore CR and Quick WMJ (1924) The scrotum as a temperature regulator for the testes American Journal of Physiology 68 70-79

Morgentaler A, Stahl BC and Yin Y (1999) Testis and temperature: a historical, clinical and research perspective Journal of Andrology 20 189-195

Mulcahy JJ (1984) Scrotal hypothermia and the infertile man Journal of Urology 132 469-470

Munkelwitz R and Gilbert BR (1998) Are boxer shorts really better? A critical analysis of the role of underwear type in male subfertility Journal of Urology 160 1329-1333

Nieschlag E, Hertle L, Fischedick A, Abshagen K and Behre HM (1998) Update on treatment of varicocele: counselling as effective as occlusion of the vena spermatica Human Reproduction 13 2147-2150
Oldereid NB, Rui H and Purvis K (1992) Life styles of men in barren couples and their relationship to sperm quality International Journal of Fertility 37 343-349

Parazzini F, Marchini M, Luchini L, Tozzi L, Mezzopane R and Fedele L (1995) Tight underpants and trousers, and risk of dyspermia International Journal of Andrology 18 125-128

Procopé BJ (1965) Effect of repeated increase of body temperature on human sperm cells International Journal of Fertility $10333-339$

Robinson D and Rock J (1967) Intrascrotal hyperthermia induced by scrotal insulation: effect on spermatogenesis Obstetrics and Gynecology 29 217-223

Robinson D, Rock J and Menkin JF (1968) Control of human spermatogenesis by induced changes in intrascrotal temperature Journal of the American Medical Association 204 290-297

Rock J and Robinson D (1965) Effect of induced intrascrotal hyperthermia on testicular function in man American Journal of Obstetrics and Gynecology 93 793-801

Saikhun J, Kitiyanant Y, Vanadurongwan V and Pavasuthipaisit K (1998) Effects of sauna on sperm movement characteristics of normal men measured by computer-assisted sperm analysis International Journal of Andrology 21 358-363

Sas M and Szöllosi J (1979) Impaired spermiogenesis as a common finding among professional drivers Archives of Andrology 3 57-60

Setchell BP (1998) The Parkes lecture. Heat and the testis Journal of Reproduction and Fertility 114 179-194

Shafik A (1991) Testicular suspension as a method of male contraception: technique and results Advances in Contraceptive Delivery Systems 7 269-279

Thonneau P, Ducot B, Bujan L, Mieusset R and Spira A (1997) Effect of male occupational heat exposure on time to pregnancy International Journal of Andrology 20 274-278

Thonneau P, Bujan L, Multigner L and Mieusset R (1998) Occupational heat exposure and male fertility: a review Human Reproduction $\mathbf{1 3}$ $2122-2125$

Tiemessen CHJ, Evers JLH and Bots RSGM (1996) Tight-fitting underwear and sperm quality Lancet 347 1844-1845

Wang C, Berman N, McDonald V, Hull L, Leung A, Swerdloff RS and Superlano L (1997) Effect of increased scrotal temperature on sperm production in normal men Fertility and Sterility 68 334-339

World Health Organization (1992) WHO Laboratory Manual for the Examination of Human Semen and Semen-Cervical Mucus Interaction 3rd Edn Cambridge University Press, Cambridge

Yamaguchi M, Sakatoku J and Takihara H (1989) The application of intrascrotal deep body temperature measurement for the noninvasive diagnosis of varicocele Fertility and Sterility 52 295-301

Zorgniotti AW and Sealfon Al (1988) Measurement of intrascrotal temperature in normal and subfertile men Journal of Reproduction and Fertility 82 563-566

Zorgniotti AW, Sealfon AI and Toth A (1980) Chronic scrotal hypothermia as a treatment for poor semen Lancet 1 904-906

Zorgniotti AW, Sealfon Al and Toth A (1982) Further clinical experience with testis hypothermia for infertility due to poor semen Urology $\mathbf{1 9}$ 636-640

Zorgniotti AW, Cohen MS and Sealfon Al (1986) Chronic scrotal hypothermia: results in 90 infertile couples Journal of Urology $\mathbf{1 3 5}$ 944-947

Received 15 September 2000.

First decision 20 October 2000

Accepted 17 November 2000. 